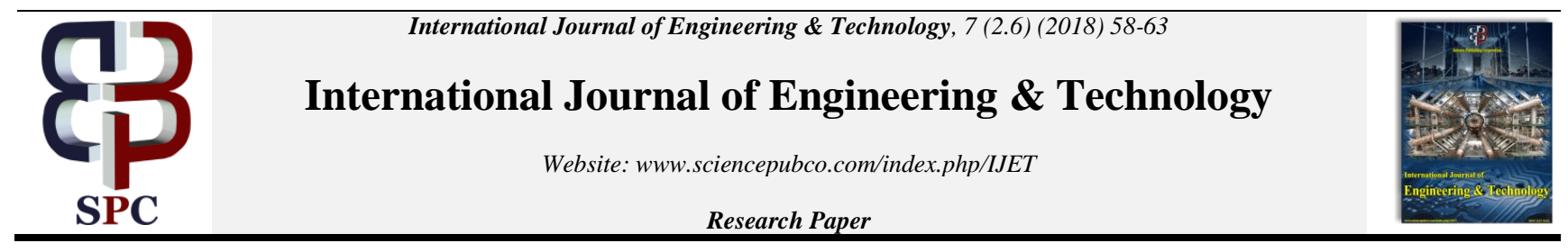

\title{
A hybrid active queue management technique for QoS based unicasting protocols in MANETs
}

\author{
Susrita Mahapatro ${ }^{1 *}$, Prabhat Kumar sahuํ, Pradeep Kumar Dash ${ }^{1}$ \\ ${ }^{1}$ Department of Computer Science \& Engineering, Siksha 'O' Anusandhan Deemed to be University, Bhubaneswar, INDIA \\ *Corresponding author E-mail: susritamahapatro@gmail.com
}

\begin{abstract}
Mobile Ad hoc Networks (MANETs) is the interconnection of several self-energy dependent mobile nodes. It is one of the selfconfigured and infrastructure less network. In MANET the issues are security, Quality Of Services, Congestion etc. The major issue of MANET is to control the congestion. Congestion means the network capacity is larger than the no packets transmitted across the network. In this paper, an effort has been made to evaluate and analyze the performance of the network by means of comparing two ondemand reactive routing protocols such as AODV [Ad-hoc On-demand Distance Vector] and DSR [Dynamic Source Routing] with each other along with also by comparing with DSDV [Destination Sequenced Distance Vector], which is a proactive routing protocol. The comparison is also being made under the light of two queue management techniques such as RED (Random Early Detection) and Droptail. A gateway discovery algorithm is being used by both AODV and DSR routing protocols, which uses the mechanism of connecting mobile nodes through gateway, however Bellman-Ford algorithm is being used by DSDV which uses the concept of including freedom from loops in the routing table. By means of simulation through NS-2 network simulator by varying the number of nodes, we observe that the performance differentials of AODV, DSR, and DSDV routing protocols are directed to foremost performance differentials to guarantee QoS for both of these protocols in RED and Droptail queue management techniques. There is a lot of queue management approach to minimize the congestion rate and transfer the data successfully to the destination. Two queue management techniques popularly used to manage queues in MANets are Active queue management and Passive queue management. Active queue management is widely used and RED is the most popular approach which gives low average delay, high throughput and lower packet drop rate. But RED algorithms have some drawback. So here we are using a hybrid approach, named as MRED by means of combining the features of both RED and ECN to overcome the drawbacks of RED.
\end{abstract}

Keywords MANETs; AODV; DSR; DSDV; RED; Droptail; MRED

\section{Introduction}

Mobile ad-hoc network, as the name suggest, an interconnection of multiple mobile nodes working over a wireless and, has selfconfiguring unstructured network. Mobility is the key feature of this type of network as they can move freely, randomly but within a specific communication range. Communication is based on packet switching, although unreliable but rapid in communication. Every mobile node in this communication system can both send and receive data packets [1]. Wireless network is basically of 2 types- Infrastructure Network and Infrastructure less network. Infrastructure Network needs a central access to connect the devices to communication among themselves, whereas Infrastructure less Network is known as a "Peer-to-Peer" Network. No centralized authority is needed for the inter communication. There is a direct communication between source and destination takes place in MANET. But if due to any reason the direct communication is failed it uses multi-hop technique. MANET follows packet switching network in which the packet will reach to the destination by any means by changing its path, if there is any problem arrive or congestion will happen. By changing the path the packet will reach to the destination. So to identify the path in the network, packet routing is required. MANET follows Proactive, Reactive and Hybrid routing techniques to send and deliver data packets throughout the network [2]. As shown in the figure 1, three nodes disorderly form an ad hoc network where the outer most nodes are not within the transmitter range to each other. During packet transmission, the intermediate node acts as a router in between these outer most nodes. There are some major challenges in ad hoc network they are-No central controlling authority, Limited Power Resource and Maintaining the Address. In no central controlling authority every mobile node is independent in interconnection; every node can communicate with another directly without the interference of the third party node. In Limited Power Resource each mobile node carries a limited energy source inform of the batteries, as a battery has its own energy capacity hence the mobile device can only be operate till the available electric source or in simple terms till that period the battery is eligible to provide the power to the device. In Maintaining the Address communication is possible only when a proper address is being maintained with the source. It is a challenge for the mobile nodes to maintain and update the address and the address parameters of a continuously moving infrastructure less system. MANET is used for safety, Traffic information. Americans are using MANET for marketing communication of vehicles and for sales communication. As shown in figure 2 , every node in the network can be viewed as an abstract entity consisting of a router and a set of associated mobile hosts. In Europe, ad-hoc network system is being designed and utilized for intercommunication among different vehicles. A setup 
for network transmission, being utilized by ad-hoc network system, can be found in almost every vehicle. They intercommunicate with each other using the same system. This helps them to know the upcoming traffic status, important local news and regarding any type of adverse situation [3].

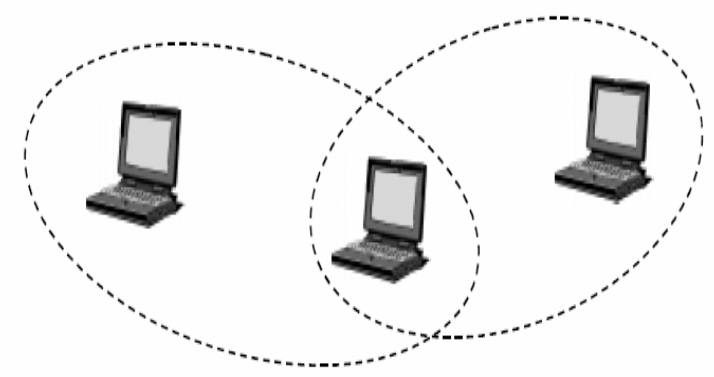

Figure 1: Example of a simple ad-hoc network with three participating nodes

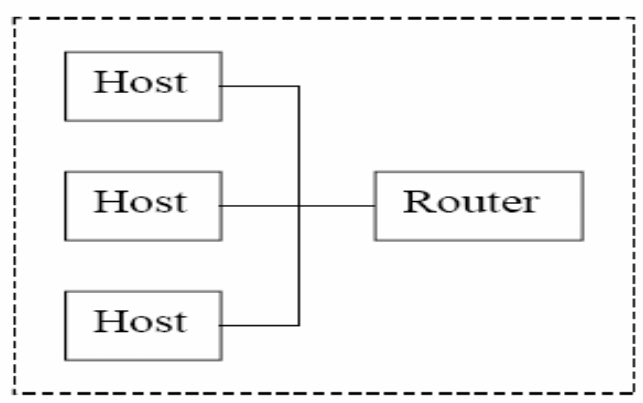

Figure 2: Block diagram of a mobile node acting as both as host and as router

Security, routing, minimize energy consumption are the main issues in MANET. Network coding is the solution for energy consumption. In this the intermediate nodes mix with the incoming data. That two data are combining together and transmit to the neighbouring node. XOR operation is performed in network coding. For consuming low amount of energy MANET security solution is used. There are many security goals but the three basic security goals are-Confidentiality, Integrity, Authentication. In confidentiality we need to protect our confidential information by using encryption method. In Integrity we have to protect the data from modification, insertion, deletion and replying by an adversary by using hash function of cryptography along with some encryption technique. Authentication service provides authentication of the party at the other end of the link, it provides hash function, encryption, digital signature and certificates etc [4].

In MANETs [5], AODV can be implemented of both unicast and multicast routing in order to discover path from a source to destination as and when necessary. Since AODV works as ondemand basis, it does not keep any additional route between sources to destination which is not in use. So the network traffic is minimum in AODV. The reactive routing protocols, AODV and DSR establish route when demanded from the source node to the destination node. A source routing instead of routing table is being used in DSR [6,7] which works by maintaining the information of each intermediate node. The Bellman-Ford routing algorithm is modified and used in DSDV [8]. The modification in this case is to overcome the drawbacks arise due to poor looping property of RIP in case of broken link. This modification resulted in increase the suitability of application of DSDV in MANETs.

\section{Related Work}

For MANETS, researchers have done much quantitative as well as qualitative analysis on different queue management techniques by using different simulators. Sahil Kochher [9] conducted an analysis of different queue management techniques that are RED, REM, BLUE, SFQ algorithm based on delay, packet loss, congestion window and throughput metric. He observed that RED and REM queue management technique is suitable and perform better in case of delay sensitive network to avoid congestion and packet loss. P.T. Mahida [10] conducted an analysis of popular queue management technique and then compares them using various parameters. He observed that REM has minimum packet drop. SFQ has highest packet drop, for end to end delay RED achieved best result. Simulation measured that single queue management is not sufficient in terms of all the parameter. Kinjal Vaghela[11] conducted an analysis of Droptail and RED then he found RED is better but sometime it is worse than Droptail in sense of packet dropping. For the better result he used modified RED i.e.TRED which calculates the packet dropping in different load. He observed that when he implemented TRED with ECN it gives less congestion in sense of packet drop. Madhuri and D.Lalkiya conducted an analysis of RED which is best but it forcefully drop all packet even the queue is empty. To overcome from this she implemented TRED which overcome all the drawback of RED and increases the throughput. She observed TRED scheme with ECN is effective than TRED and RED without ECN. Sarita Simaiya, Anurag Shrivastava, Narayan Prasad keer[12] conducted an analysis of RED and IRED based on congestion and packet loss and implemented improved RED (IRED) to decide the dropping probability of packet to minimize the effect of network congestion. He observed IRED gives lower packet loss rate as compare to RED.

\section{Quality of Service in MANETs}

Quality of service [3] refers to the efficiency of the network to transmit the data in terms of speed, time and drop. It includes a secure a defective route of transmission. Bandwidth is the vital component for control of QoS [13]. To improve the QoS, the available bandwidth can be used in 2 ways-To analyze network performance, to optimize end to end performance. In MANET it is a challenging job to maintain the quality of service due to the infrastructure less and continuously moving mobile nodes. The factors which influence the quality of service are Low Throughput, Packet Drop, errors in data packets due to noise or other intervening factors. There is no particular standard has been accepted worldwide to measure the standard of quality in an Ad-hoc network [14].

\section{Congestion}

Every network has its own network capacity and in Ad-hoc network data are transmitted mostly in terms of data packets. When the number of data packets more than the capacity of the network tries to transmit over the network then congestion in the network occurs. The Ad-hoc network system is designed in such a way that a single node can act as a sender or receiver along with an intermediate node that means when the receiver is not in the range of the sender than another node(s) in between acts as the middle node to transmit the data packets. Hence it suffers from the load for itself along with the load to transmit the data for other nodes which makes the congestion scenario worst. However in the same time other nodes sit ideal. It creates a major challenge for the effective resource utilization. The load on the network can also be increased due to the retransmission of data. Let's assume a data packet is corrupt after travelling four intermediate nodes. As the data for the receiver is useless sender has to retransmit the same data to the receiver which ultimately increase the network load. Congestion creates long delay in transmission of data, High overhead, large packet drops to overcome this type of situation [15]. 


\section{Queue Management}

Queue management is one of the best methods to avoid congestion Queuing technique used for ordering MANETS in a queue. In queuing technique resources are divided into packets and they are served in order. It controls the transmission of packet by deciding which packet should be transmitted into the queue of the receiving node and which packet should be dropped at the tail side. There are so many queuing techniques available in MANET. This technique is mostly used for congestion avoidance. The queuing techniques are First in First out (FIFO), Last in First out (LIFO), Priority Queue (PQ), Servicing Random Order, Round Robin and Drop tail Queue etc. Drop tail, RED, REM are most frequently used for congestion avoidance [16].

\subsection{Classification of Queue Management technique}

We know that each \& every node in the network maintains a set of queues that use to hold data packets within themselves. In order to avoid heavy congestion and network load in MANET we use two queue management techniques.

Passive queue management: It is very easy to implement because it has less overhead computational overhead. This algorithm knows about the buffer status when packet drop occur [8]. Example: Droptail, SFQ, DEC bit

Droptail queue: Most of the mobile devices are using drop tail. In Drop Tail Queue packets which entered into the queue first is delivered first. It uses FIFO policy. It allows the data packets to enter into the front end of the queue till the queue is empty, i.e till it has the capacity of holding at least one data packet in the queue of the receiving node. When the queue becomes full it drop packets from several nodes. In drop tail queue management after the queue get full all packets from several links are dropped and it compresses the congestion window. The connection suffers from problem like Global Synchronization which is the major disadvantage of Drop tail queue. Random early detection avoids the congestion in network better than drop tail queue management.

Active queue management: Active queue Management used to prevent global synchronization. The major disadvantage of the Passive queue management technique is it does not have any mechanism to detect congestion at least to that point of time when a single data packet is dropped. Packets by using passive queue management technique, drops when the queue is full, is not a full phase solution for congestion and lacks in quality of service significantly. The active queue management technique uses a unique technique. It uses the concept of control as well as prevention. In this technique before the queue become full, when it get the notification about potential congestion that may happen in the near future, slow down the data transfer rate and in the worst case only it drops packets. This technique helps it to prevent queue overflowing and hence this approach is known as active queue management [8].Example: Random Early Detection (RED), Random Exponential Marking (REM)

RED: In MANETs congestion can occur at any time depending on the number of data packets to be transmitted and the capacity of the node[18]. When the number of incoming packets to the receiver node is more than the capacity of the buffer, then transmission stops. So the transmission path becomes congested amounting to packet loss. RED is one technique which detects congestion before congestion of network happen. It was developed by Floyd in year 1993 for reducing the packet loss and delay. RED maintains an average queue length to detect the congestion. This algorithm uses a technique to calculate the packet dropping probability basing on the average queue length (Avg_Q_Len). When the queue is empty that is does not contain any packets it is represented by Min_Len, likewise when it is full it is represented by Max_Len. The transmission of data packets depends on the Avg_Q_Len, Max_Len and Min_Len. The dropping probability is calculated when Avg_Q_Len is in between Max_Len and Min_Len. Packets dropped when Avg_Q_Len is higher than Max_Len, otherwise packet is allowed to enter into the queue of the receiving node. However when the Avg_Q_Len is closed to Max_Len an exclusive congestion notification is sent to the source to direct to slow down the data transfer rate. Likewise when Avg_Q_Len is closer to Min_Len, a notification is sent to increase the sending data rate.

$E C N$ : ECN stands for Explicit Congestion Notification. This mechanism develops a strategy to mark packets to notify the source and destination regarding the status of the data packets as well as the environmental conditions like congestion, broken links etc. This mechanism helps to minimize the data drop using explicit marking. We in our research paper analyzed the application of ECN and studies the performance metrics using RED (Random Early Detection) and named it as MRED. The mechanism used in MRED is the collaboration of RED algorithm with ECN marking techniques. It marks the packets which have high probability of drop. Hence it has better performance in respect of End to End delay, packet drops and network performance. This technique is simple. It requires an ECN field in the data packets which consumes only two bits, named as ECN-Capable Transport (ECT) Bit and Congestion Experienced (CE) bit. The ECT bit can only be set and modified by the source to broadcast and check the destination node is ECN capable or not. The CE bit can be set and modified by any intermediate mobile nodes to indicate congestion in between the transportation path.

\section{MRED- A Hybrid Queue Management Al- gorithm}

\section{CONGESTION NOTIFICATION ALGORITHM:}

\section{STEP: 1 - START}

STEP: 2- Check whether ECT(0) or ECT(1) are active or not. if (not active)

Suspend the transmission process and Print "Error: Receiver not capable of ECN"

else

Set $\mathrm{ECHO}=1$

STEP: 3- Set Min $=25 \%$ of Queue Size of the Receiver$\mathrm{Max}=\mathrm{Min} * 3$ and $\mathrm{Wq}=0.002$

STEP: 4- Calculate Average Queue Length as avg= (1$\mathrm{Wq}) * \operatorname{avg}+\mathrm{Wq} * \mathrm{qe} / / \mathrm{avg}$ is the previous queue size

STEP: 5- if (avg<min)

Then Enqueue Packet.

STEP: 6- if $(\min <$ avg $<\max )$

Then Calculate $\mathrm{Tl}=(\max -\min ) / 3$

if $(\min <$ avg $<\min +\mathrm{Tl})$

Then Calculate $\mathrm{P} 1=($ avg-min $) /(\max -\min ) 3$

Send message to the sender to increase sending rate if packets available.

if $(\min +\mathrm{Tl}<\mathrm{avg}<\min +2 * \mathrm{Tl})$

Then Calculate $\mathrm{P} 1=($ avg-min/max-min $)$

if (P1 is low)

Then Enqueue Packet

else

Calculate $\mathrm{Pa}=\mathrm{Calcp}$ (P1, flow_id_packet)

if ( $\mathrm{Pa}$ is low)

Then Enqueue Packet

else

Enqueue Packet and Set CE field ip packets cate the sender that loads is minimum.

if $(\min +2 * \mathrm{Tl}<\operatorname{avg}<\max )$

then

Calculate $\mathrm{P} 1=[\operatorname{avg}-\min / \max -\min ]+\max$

Calculate $\mathrm{Pm}=\mathrm{Calcp}(\mathrm{P} 1$, flow_id_packet $)$

if ( $\mathrm{Pm}$ is low) 


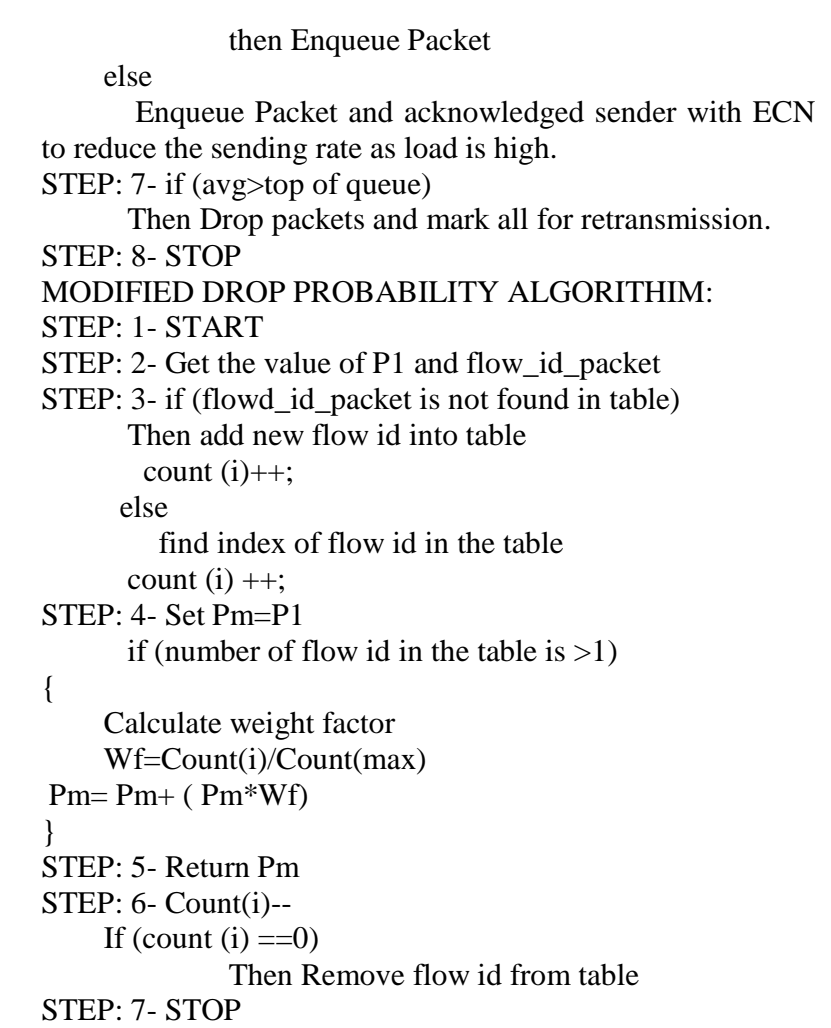

Terms Used In The Algorithm:-

ECT: ECN capable Transport

ECN/CE: Congestion Experienced bit in IP header 3

ECHO: ECHO bit in TCP header

Max: Maximum Threshold

Min: Minimum Threshold

Wq: Weight Queue Constant

avg: Average Queue Size

qe: Instantaneous queue size.

P1: Drop Probability of Packet loss

Pm: Modified drop Probability

i: index of flow id in the table

Wf: Weight factor

\section{Performance analysis and simulation work}

The purpose of this work is to determine the best routing protocol by comparing three routing protocols such as AODV, DSR and DSDV by analyzing the QoS simulation Parameters, as provided in Table 1, implemented for wireless communication in MANETs The study and evaluation in the current study has been carried out by exhaustive literature review along with real time simulation. There are four types of quantitative performance matrices used for this study namely, throughput, end-to-end delay, normalized over heads and packet delivery ratio. The performance of a network [17] is measured in terms of certain factors. These factors describe the scalability, robustness and efficiency of the network. The following four quantitative performance parameters have been used for this study:

A. Throughput: Throughput of a network is the average number of data packets transmitted from the source to the destination in a unit time. Throughput of the network is measured by Kilobits per second.

B. Packet-delivery ratio: The Packet Delivery Ratio or PDR of a network is calculated by calculating the divisional ratio of total number of data packets successfully delivered to the destina- tion node from the source node with the total number of data packets which are being sent by the sender to the destination node. The ratio will be 1 if there will be no packet loss and it is in case of the ideal situation.

$C$. Normalized routing overhead: Normalized Routing Overhead of a node is calculated by finding out the divisional ration of total number of data packets sent to the next node with the total number of data packets received from the previous nodes which includes the received data packets as an intermediate node as well as a destination node.

D. Average end-to-end delay: The end to end delay for a single data packet is measure as the total time taken for a single data packet to reach at the destination node from the source node irrespective of packet loss. The average end to end delay is calculated by finding the ration of the sum of the delays with the number of the sent data packets. The end to end delay is measure by Milliseconds.

Table 1: Simulation Parameters with values

\begin{tabular}{cc} 
Table 1: Simulation Parameters with values \\
\hline Parameters & Values \\
\hline & \\
Protocols & AODV, DSR, DSDV \\
Number of Nodes & $10,20,30,40,50$ \\
Simulation & 150 Sec \\
Environment & Omni Directional \\
Traffic & TCP \\
Packet Size & 512 Bytes \\
Mobility & Random Waypoint \\
Simulator & NS2 \\
\hline
\end{tabular}

From the simulation results in Figure 3, it is observed that the packet-delivery ratio unevenly increases with increasing the number of nodes for Droptail and RED queue management. Since the nodes are set at random motion, it may so happen that, the network congestion at a particular instant of time is low (i.e. when nodes are away from each-other) \& at another instant of time, the congestion is high. This explains the uneven fall of packetdelivery ratio with the increase in the number of nodes. The Endto-End delay shown in Figure 4 reveals that, higher node density increases the number of neighbouring nodes and that consequently increases the congestion thereby increasing the delay for Droptail and RED queue management techniques. Since the number of nodes was increased, the AODV, DSR and DSDV protocol behaves more pro-actively rather than reactive. As a result of which the average end-to-end delay increases which portrays the fact that with the increase in the network congestion, the transmission of data packets suffer from different delays in the network.

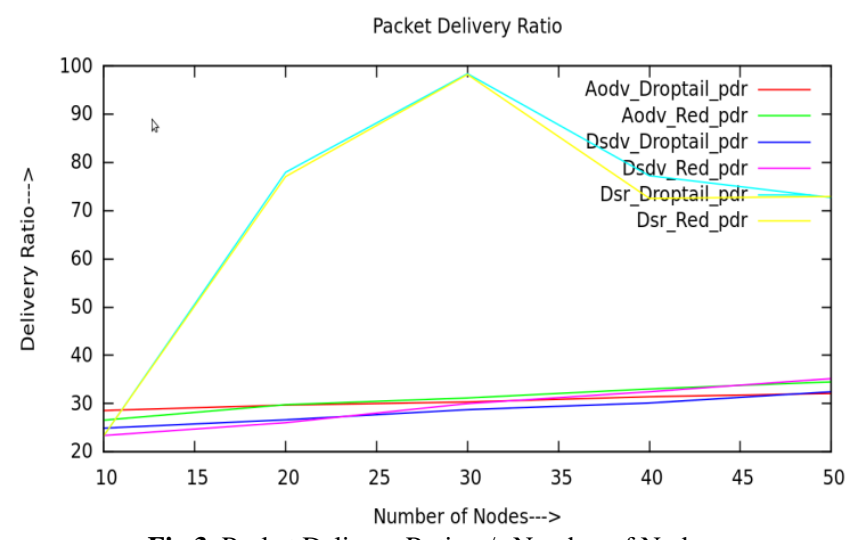

Fig.3. Packet Delivery Ratio v/s Number of Nodes 


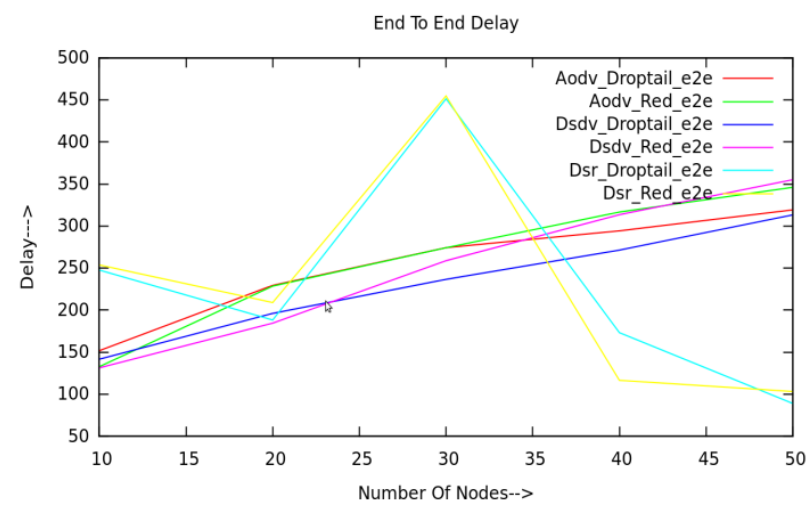

Fig.4.Delay v/s Number of Nodes

In Figure 5, the normalized routing overhead is plotted under different number of nodes for Droptail and RED queue management approaches. It is observed that the overhead increases unevenly with increasing number of nodes for AODV, DSR and DSDV protocols. Since the number of nodes goes on increasing, the congestion decreases accordingly which results in larger number of data packets received as compared to the number of packets sent or forwarded, which explains the increasing nature of our graph.

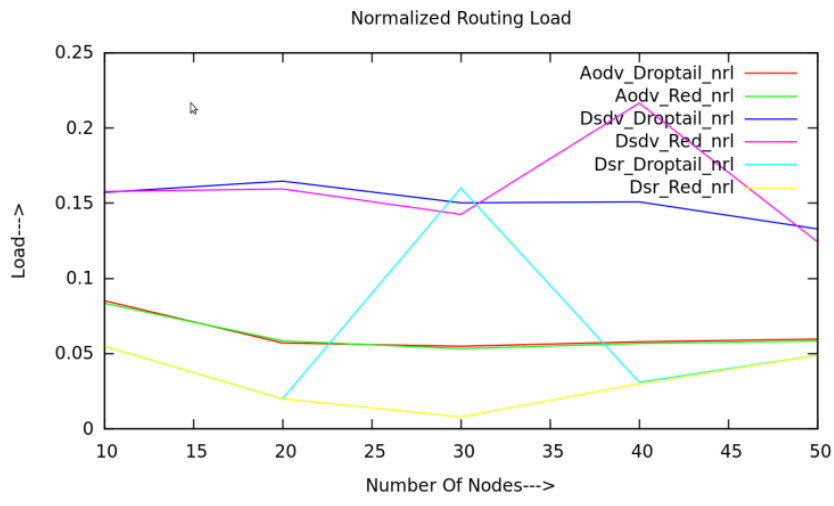

Fig.5. NRL v/s Number of Nodes

From the simulation results in Figure 6, it is observed that the throughput increases with increasing number of nodes for AODV, DSR and DSDV protocols. Since the z number of nodes goes on increasing, the number of bits sent or received also increases which explains the increase of throughput.

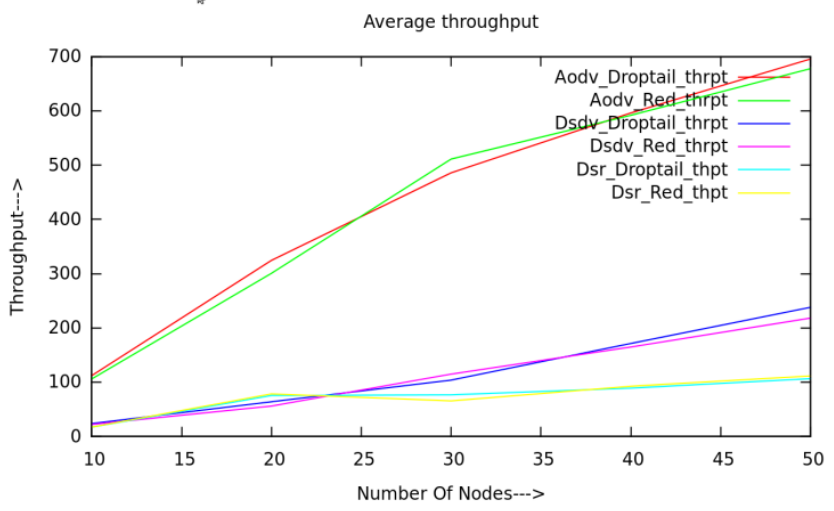

Fig.6. Throughput v/s Number of Nodes

\section{Conclusion}

We have conferred a performance comparison of on-demand reactive routing protocols, namely, AODV and DSR as well as one pro-active routing protocol DSDV for MANETs using Droptail and RED queue management techniques. Through our paper, we discussed the several features of the mobile ad hoc networks and evaluate the performance of AODV,DSR and DSDV by considering the node density, which was changed with the change of number of nodes in a fixed area. The number of mobile nodes was varied from 10 to 50 at first as 10,and then to 20, 30, 40 and lastly to 50. We also implemented the AODV, DSR and DSDV routing protocols in NS2 using Droptail and MRED queue management techniques by forming a typical simulation environment with different performance parameters such as end-to-end delay, packet delivery ratio, average throughput and normalized routing overheads. Our simulation results show that the throughput and the packet delivery ratio need to be increased with increasing in number of nodes. As the number of nodes is increased, the performance of AODV and DSR is significantly good in comparison to DSDV in MRED queue environment. Thus it can be observed that for a higher number of nodes, AODV and DSR proves to be a better robust protocols in comparison to DSDV with MRED queue environment in comparison to Droptail and provides better throughput, packet delivery ratio and minimizes the end to end delay as well as the normalized overhead routing.

\section{References}

[1] Asma Tuteja, Rajneesh Gujural, Sunil Thalia, "Comparative Performance Analysis of DSDV, AODV and DSR Routing Protocols in MANET using NS2", International conference on Advances in computer Engineering , 2010.

[2] Rajesh Kochher, Ritu Mehta, "Performance Analysis of Reactive AODV and DSR with Hybrid GRP Routing protocols under IEEE 802.11g MANET", IEEE conference, 2016.

[3] Amit Dangi, Mr.K K Tiwari, "A secure hybrid communication approach for disaster recovery system in MANETS: review paper", International Journal of Advance Research in Computer Science and Electronics Engineering, Vol.5, Issue 1, January 2016.

[4] Shruti Patel, Fenl Khatiwala, "A review paper of an encryption scheme using network coding for energy optimization in MANET", IEEE conference, 2016.

[5] M.S. Corson,A. Ephremides, "A Distributed Routing Algorithm for Mobile Wireless Networks", ACM J.Wireles Networks ,1(1) ,Jan 1995

[6] Md. Anisur Rahman, Md. Shohidul Islam, Alex Talevski "Performance Measurement of Various Routing Protocols in Adhoc Network", International Multi Conference of Engineers and Computer Scientists, Hong Kong, March 18 - 20, 2009.

[7] D.Loganathan, P.Ramamoorthy, "Enhanced DSR Protocol with Multicost Algorithm in Wireless Ad hoc Networks", International Journal of Engineering Science and Technology (IJEST), Vol. 4 No.08 August 2012.

[8] C.E. Perkins \& P. Bhagwat, "Highly Dynamic Destination Sequence-Vector Routing (DSDV) for Mobile Computers", Computer Communication Review, Vol-24, Issue-4,PP: 234-239, 2009.

[9] Sahil Kochher, Malti Sanghal, Rajesh Kochher, Gurnam Singh, "A review on Active and Passive Queueing Techniques", International Journal for Science Emerging Technology: pp:1622, 2014

[10]P.T.Mahida, Kinjal Patel, Nayan Vanza, Siddharth Patel, "A Comparison Analysis of Queue Management Techniques using NS-2 Simulator", International Journal of Computer Applications, volume 65-No 6,March 2013.

[11] Kinjal Vaghela, "Improved Congestion Control Using Modified RED Algorithm over MANET", International Journal of Engineering Development and Research, volume 4, Issue 2, 2016.

[12] Sarita simaiya, Anurag Shrivastava, Narayan Prasad Keer, "IRED Algorithm for improvement in performance of mobile ad hoc network", fourth International Conference on communication system and network technologies, 2014.

[13] Sahu P.K., Pattanayak B.K., "Quality of Service based Multicasting Routing Protocols for MANETs: A Survey", International Journal on Communications Antenna and Propagation (IRECAP), pp 423-438, vol-7(5), Oct-2017.

[14] Md. Anisur Rahman, Md. Shohidul Islam, Alex Talevski "Performance Measurement of Various Routing Protocols in Adhoc Network", International Multi Conference of Engineers and Computer Scientists, Hong Kong, March 18 - 20, 2009. 
[15]Rajgopal .G, Manikandan .K, Sivakumar .N, “QoS Routing using Energy Parameter in Mobile Ad Hoc Network”, International Journal of Computer Applications, Volume 22,Issue-4, May 2011.

[16] Sahu P.K., Pattanayak B.K., "Comparative Performance Analysis Of Zrp Routing Protocol For Multicasting In Manets Using Droptail And Red Queue Management", Far East Journal of Electronics and Communications, Volume 17, Issue 2, Pages 295 - 310 , April 2017.

[17] Ajay Shah, Hitesh Gupta, Mukesh Baghel, "Energy Efficient Routing Protocols in Mobile Ad hoc Networks ", International Journal of Engineering Research and Development, Volume 2, Issue 7 PP. 45-51, August 2012. 